\title{
Prevalence of Cervical Infection and Genotype Distribution of Human Papilloma Virus Among Females in Da Nang, Vietnam
}

\author{
SONG NGUYEN VAN ${ }^{1}$, MINH NGUYEN KHAC ${ }^{1}$, JAN DIMBERG $^{2}$, \\ ANDREAS MATUSSEK ${ }^{3,4,5}$ and ANNA J. HENNINGSSON ${ }^{5}$ \\ ${ }^{1}$ Department of Medical Laboratory, Da Nang University of Medical Technology and Pharmacy, Da Nang, Vietnam; \\ ${ }^{2}$ Department of Natural Science and Biomedicine, School of Health and Welfare, \\ Jönköping University, Jönköping, Sweden; \\ ${ }^{3}$ Karolinska University Laboratory, Karolinska University Hospital, Stockholm, Sweden; \\ ${ }^{4}$ Department of Laboratory Medicine, Division of Clinical Microbiology, \\ Karolinska Institute, University Hospital Huddinge, Stockholm, Sweden; \\ ${ }^{5}$ Clinical Microbiology, Division of Medical Diagnostics, Region Jönköping County, Jönköping, Sweden
}

\begin{abstract}
Aim: The goal of the present study was to determine the prevalence and distribution of high-risk human papilloma virus (HPV) genotypes in women from two districts in and around Da Nang city, Vietnam. Materials and Methods: All participants were randomly selected, 200 from the Hai Chau district and 200 from the Son Tra district. The detection and genotyping of HPV were performed by realtime polymerase chain reaction (PCR) technique. Results: Out of a total of 400 women, we found that 38 (9.5\%) were infected with a high-risk HPV genotype, the most prevalent genotypes being 16, 18, 58 and 59. By assessment of the HPV findings in relation to sociodemographic characteristics, we found significant differences between the two study districts and between the age groups, as well as differences associated with occupation and the use of contraceptives. Conclusion: The proportion of high-risk genotypes other than 16 and 18 was relatively high, and since the HPV genotype distribution is known to vary greatly across populations, the information from this study can be used for planning of screening and vaccination programs in Da Nang.
\end{abstract}

The human papillomavirus (HPV) is one of the most common causes of sexually transmitted disease in both

This article is freely accessible online.

Correspondence to: Dr. Anna J. Henningsson, Division of Medical Diagnostics, Clinical Microbiology Laboratory, County Hospital Ryhov, 55185 Jönköping, Sweden. Tel: +46 36321226, Fax: +46 36180073, e-mail: anna.jonsson.henningsson@rjl.se

Key Words: Cervical cancer, human papilloma virus, high-risk HPV genotype, prevalence, Vietnam. women and men all over the world. Studies on the etiology of cervical cancer have shown that approximately $99 \%$ of cases are associated with HPV infection $(1,2)$.

More than 200 genotypes of HPV have been identified and are generally classified as high-risk and low-risk HPV based on their association with premalignant and malignant lesions (3). The high-risk types $16,18,31,33,35,39,45,51,52,56$, 58 and 59 have been detected in $99 \%$ of all cervical cancer, with approximately $70 \%$ of all invasive cervical cancer cases being due to HPV types 16 or $18(2,4)$.

Prophylactic vaccination against HPV has been conducted in many countries for the prevention of cervical cancer. There are a number of variants such as monovalent (HPV16), bivalent (HPV16 and 18), quadrivalent (HPV6, 11, 16 and 18) and nonavalent (HPV6, 11, 16,18, 31, 33, 45, 52 and 58) vaccines $(5,6)$. In Vietnam, the measures for cervical cancer prevention include, primary screening for HPV and vaccination against HPV (7). It has been shown that there are differences in the genotype distribution in women with multiple HPV infections not only among women of different ethnicity but also women from different regions (8). Moreover, the tendency of HPV genotypes to cluster in multiply infected women has been frequently observed (8). Knowledge about the type-specific distribution and the prevalence of HPV cervical infection in women from diverse areas and ethnicity is a prerequisite for the planning of an efficient and adequate screening and vaccination strategy with the best possible outcome. Some studies have been conducted within Vietnam, especially in Ho Chi Minh City and Hanoi (7, 9-11), but also in populations from Thai Nguyen, Hue and Can Tho (9). These studies showed a prevalence of high-risk HPV infection, ranging between $2-11 \%$.

There are limited epidemiological data on the prevalence of HPV in cervical infection and distribution of HPV types 
in cohorts resident in and around the city of Da Nang, Vietnam. Therefore, the aim of the present study was to examine HPV prevalence and HPV genotype distribution, based on real-time polymerase chain reaction (PCR), in cervical swab samples from women in Da Nang represented by the Hai Chau and the Son Tra districts.

\section{Materials and Methods}

Study population and sample collection. In this study, we used cervical samples from 400 women participating in a HPV-screening program in Da Nang city in 2015. Married, non-pregnant women aged 18-49 years were included. Exclusion criteria were a) treatment for gynecological diseases/symptoms during the previous 7 days, b) on-going menstruation, c) sexual intercourse during the 3 days prior to sampling, e) psychiatric disorder. Informed consent was obtained from each participant and personal interviews were performed which included information on sociodemographic characteristics including age, occupation, education and use of contraceptives.

All participants were randomly selected, 200 from the Hai Chau district and 200 from the Son Tra district. Da Nang has a total of seven districts with 56 wards. The Hai Chau district has 13 wards and the Son Tra district has seven wards. In each district, participants between 18-49 years of age were sampled, and the probability of being selected was proportional to the size of the associated ward.

The samples were randomly selected from the list of eligible women by a computer-based program.

Ethics approval and consent to participate. Informed consent to use the results of the analysis was obtained from each of the study participants. The study was approved by the Ethics Committee of Da Nang University of Medical Technology and Pharmacy (01/QDDHKTYDDN-HDDDYS).

$H P V$ detection and typing. Cervical swab samples were dissolved in $1 \mathrm{ml}$ of $0.9 \% \mathrm{NaCl}$ and stored at $-20^{\circ} \mathrm{C}$ until analysis. Genomic DNA from $200 \mu$ of sample solution was extracted by DNA Mini Kit Invisorb ${ }^{\circledR}$ Spin Virus 0814 (Sacace, Biotechnologies, Como, Italy) according to the manufacturer's protocol. The concentration and quality of genomic DNA was checked by a NanoDrop 2000 spectrophotometer (Thermo Scientific, Columbia, USA) and 1\% agarose gel electrophoresis.

HPV DNA genotypes were identified by real-time PCR using 14 HPV Genotypes Real-TM Quant Kit (Sacace Biotechnologies) for 14 high-risk types (namely types 16, 18, 31, 33, 35, 39, 45, 51, 52, $56,58,59,66$ and 68) and analyzed on Stratagene Mx3000P system (Stratagene, San Diego, CA, USA). All processed samples were analyzed at the Laboratory of Molecular Biology, Dan Nang University of Medical Technology and Pharmacy.

Statistical analyses. In order to achieve high statistical power, the sample size was calculated according to Charan et al. (12). With the estimated prevalence of HPV infection in women in the community being about $12 \%$, the required minimum sample size was calculated to be approximately 163 under the conditions of 5\% Type 1 error and the reliability of the study $95 \%$.

Differences in the prevalence of HPV DNA between the groups were analyzed using the chi-squared test. Statistical analysis was performed using SPSS Statistics software (version 19, 2012; SPSS, Chicago, IL, USA). Results were considered significant at $p<0.05$.

\section{Results}

Out of a total of 400 women, we found that 38 were infected with a high-risk HPV genotype, thereby exhibiting an overall prevalence of $9.5 \%$. Sociodemographic characteristics and the prevalence of high-risk HPV infection of the study subjects are presented in Table I. Thirteen different HPV types were detected among the $38 \mathrm{HPV}$-positive women, the most prevalent types being 16, 18, 58 and 59 (Table II).

Single HPV type infection was detected in $24 \mathrm{HPV}$ positive cases $(63.1 \%)$ and multiple infection with two, three and four HPV types was detected in $26.3 \%, 5.3 \%$ and $5.3 \%$, respectively.

By assessment of the HPV findings in relation to demographic characteristics, we found significant differences between the two study districts and between age groups, as well as differences associated with occupation and the use of contraceptives. However, we found no association between HPV prevalence and educational level (Table I). When we analyzed the age-specific prevalence we found significantly higher HPV prevalence in women aged 18-29 years compared to women $30-39$ years $(p<0.001)$ and women 40 49 years $(p<0.006)$, respectively.

\section{Discussion}

In this study, we investigated a broad spectrum of 14 highrisk HPV genotypes, including the most common types of HPV 16 and 18, and found 13 high-risk types among the cervical samples collected from women in Da Nang city. We report a prevalence of $9.5 \%$ of cervical HPV infection in Da Nang city, which is at the upper end of range of $2 \%$ to $11 \%$ previously reported from other cities in Vietnam $(7,9-11)$. Considering the Hai Chau district, a downtown area with offices, schools, amusement parks and business centers, the prevalence $(15.0 \%)$ of HPV infection was found to be significantly higher in comparison with women in the Son Tra District (4.0\%), which is a new developing area located of the coast and dominated by fishermen, ordinary workers and tourism.

As expected, we found that the most common genotypes HPV 16 and HPV 18 dominated among the 38 HPV-positive women in Da Nang, a finding that is similar to previous results reported from other places in Vietnam (10) and in other countries $(4,13,14)$. About half of the $38 \mathrm{HPV}$ positive women in Da Nang were infected with only HPV 16 or 18 , or both, and the rest with other risk types of HPV. This finding corroborates a previous study of women in the nearby city of Hue (7). Moreover, our data indicate a high prevalence of HPV59 in comparison to earlier reports from 
Table I. Demographic characteristics and prevalence of cervical high-risk human papilloma virus (HPV) infection in the study group of 400 women in Da Nang, Vietnam.

\begin{tabular}{|c|c|c|c|c|c|}
\hline \multirow[b]{2}{*}{ Factor } & \multirow[b]{2}{*}{ Subgroup } & \multirow[b]{2}{*}{ No. of cases } & \multicolumn{2}{|c|}{ Prevalence, n (\%) } & \multirow[b]{2}{*}{$p$-Value } \\
\hline & & & HPV-positive & HPV-negative & \\
\hline \multirow[t]{2}{*}{ District } & Hai Chau & 200 & $30(15.0)$ & $170(85.0)$ & \multirow[t]{2}{*}{$<0.001$} \\
\hline & Son Tra & 200 & $8(4.0)$ & $192(96.0)$ & \\
\hline \multirow[t]{3}{*}{ Age (years) } & $18-29$ & 92 & $20(21.7)$ & $72(78.3)$ & \multirow[t]{3}{*}{$<0.001$} \\
\hline & $30-39$ & 188 & $14(7.4)$ & $174(92.6)$ & \\
\hline & $40-49$ & 120 & $4(3.3)$ & $116(96.7)$ & \\
\hline \multirow[t]{2}{*}{ Occupation } & Employed & 278 & $20(7.2)$ & $258(92.8)$ & \multirow[t]{2}{*}{0.017} \\
\hline & Unemployed & 122 & $18(14.8)$ & $104(85.2)$ & \\
\hline \multirow[t]{2}{*}{ Education } & High school or less & 238 & $22(9.2)$ & $216(90.8)$ & \multirow[t]{2}{*}{0.832} \\
\hline & College degree or higher & 162 & $16(9.9)$ & $146(90.1)$ & \\
\hline \multirow[t]{2}{*}{ Condom use } & Yes & 294 & $22(7.5)$ & $272(92.5)$ & \multirow[t]{2}{*}{0.022} \\
\hline & No & 106 & $16(15.1)$ & $90(84.9)$ & \\
\hline
\end{tabular}

Vietnam (9). Thus, we found a high prevalence of HPV genotypes other than 16 and 18 , and other studies have shown that the genotype distribution varies considerably across populations worldwide (15). This reflects the importance of knowing the regional genotype-specific distribution and the prevalence of HPV cervical infection in women when designing screening programs and planning vaccination strategies. Moreover, these findings are important for the assessment of the impact of intervention models with the purpose to control cervical cancer incidence.

In a substantial percentage of HPV infections, two or more genotypes may be found and $20-50 \%$ of the infected women carry more than one genotype (16). Our study revealed a prevalence of $36.9 \%$ of infection with multiple high-risk HPV types in cervical samples from the $38 \mathrm{HPV}$-positive women in Da Nang. Pham et al. demonstrated $41.6 \%$ and $20.0 \%$ prevalence of multiple-type HPV infection in HPVpositive women in Ho Chi Minh City and Hanoi, respectively (10). It has been shown that there are differences in the genotype distribution in women infected with multiple HPV types between women from different regions (8) Whether our findings reflect such differences must be investigated in a larger number of HPV-positive women.

Our data about the age-specific prevalence of HPV infection among women in Da Nang show a significant decline related to higher age. This is consistent with another study from Ho Chi Minh City (10). The results may be explained by a dynamic population, especially among the young in Da Nang, in combination with sexual transmission during the start of sexual activity. HPV acquisition is generally more common in younger women with multiple sexual partners and many HPV infections are transient (17-19).
Table II. High-risk human papilloma virus (HPV) genotypes detected in cervical samples from $38 \mathrm{HPV}$-positive women in the study group of women in Da Nang, Vietnam.

\begin{tabular}{lcc}
\hline Genotype & No. of cases & Prevalence $(\%)$ \\
\hline 16 & 12 & 31.6 \\
18 & 8 & 21.1 \\
31 & 2 & 5.3 \\
33 & 2 & 5.3 \\
35 & 4 & 10.5 \\
45 & 2 & 5.3 \\
51 & 4 & 10.5 \\
52 & 4 & 10.5 \\
56 & 4 & 10.5 \\
58 & 6 & 15.8 \\
59 & 6 & 15.8 \\
66 & 2 & 5.3 \\
68 & 2 & 5.3 \\
\hline
\end{tabular}

Studies have suggested that the use of condoms has little, if any, protective effect against HPV infection $(20,21)$. However, our study showed that condom use was associated with a significantly lower prevalence of high-risk type HPV, which was also previously reported from other populations $(11,22,23)$.

We found a significantly higher prevalence of HPV infection in unemployed women compared to employed women. However, the HPV prevalence was not associated with the level of education. Interpretation of these data is delicate, but there may be connections with lifestyle and sexual behavior. Other studies have also found somewhat contradictory results and indications of gaps existing between women's knowledge and practice. Ramamurthi et 
$a l$. found for example that the awareness of cervical cancer, available screening tests and vaccines was higher among employed women than among unemployed, but that unemployed women were more likely to adhere to screening and vaccination programs once provided with the appropriate information (24).

\section{Conclusion}

We found an overall HPV prevalence of 9.5\% in Da Nang city, but also significant differences between the two study districts that could possibly be associated with socioeconomic factors. The proportion of high-risk genotypes other than 16 and 18 was relatively high, and since the HPV genotype distribution is known to vary greatly across populations worldwide, it is important to take regional data into consideration in the planning of screening and vaccination programs. In the future, additional studies are warranted, using larger number of samples, in order to confirm the HPV prevalence and distribution in this study and also to relate the data to the incidence of cervical cancer.

\section{Competing Interests}

The Authors declare that they have no competing interests.

\section{Funding}

This work was supported by grants from Da Nang University of Medical Technology and Pharmacy.

\section{Acknowledgements}

The Authors thank Hai Chau Centre Medical Diagnostic and Son Tra Centre Medical Diagnostic for sample collection. We also thank the participants for providing personal information and samples for this study.

\section{References}

1 World Health Organization: Cervical Cancer, Human Papillomavirus (HPV) and HPV vaccines. Key Points for Policymakers and Health Professionals. Geneva, World Health Organization, 2008.

2 Walboomers JM, Jacobs MV, Manos MM, Bosch FX, Kummer JA, Shah KV, Snijders PJ, Peto J, Meijer CJ and Munoz N: Human papillomavirus is a necessary cause of invasive cervical cancer worldwide. J Pathol 189: 12-19,1999.

3 Munoz N, Bosch FX, de Sanjose S, Herrero R, Castellsague X, Shah KV, Sneijders PJ and Meijer CJ: Epidemiologic classification of human papillomavirus types associated with cervical cancer. N Engl J Med 348: 518-527, 2003.

4 Smith JS, Lindsay L. Hoots B, Keys J, Franceschi S, Winer R and Clifford GM: Human papillomavirus type distribution in invasive cancer and high-grade cervical lesions: a meta-analysis update. Int J Cancer 121: 621-632, 2007.
5 Lu B, Kumar A, Castellsague X and Giuliano AR: Efficacy and safety of prophylactic vaccines against cervical HPV infection and diseases among women: a systematic review and metaanalysis. BMC Infect Dis 11: 13, 2011.

6 Wagner M, Bennetts L, Patel H, Welner S, de Sanjose S and Weiss TW: Global availability of data on HPV genotype distribution in cervical, vulvar and vaginal disease and genotypespecific prevalence and incidence of HPV infection in females. Infect Agent Cancer 10: 13, 2015.

7 Vu LT, Bui D and Le HT: Prevalence of cervical infection with HPV type 16 and 18 in Vietnam: implications for vaccine campaign. BMC Cancer 13: 53, 2013.

8 Vaccarella S, Franceschi S, Snijders PJ, Herrero R, Meijer CJ, Plummer M and IARC HPV Prevalence Surveys Study Group: Concurrent infection with multiple human papillomavirus types: pooled analysis of the IARC HPV Prevalence Surveys. Cancer Epidemiol Biomarkers Prev 19: 503-510, 2010.

$9 \mathrm{Vu}$ TH L and Bui D: Prevalence of cervical HPV infection among married women in Vietnam 2011. Asian Pac J Cancer Prev 13: 37-40, 2012.

10 Pham TH, Nguyen TH, Herrero R, Vaccarella S, Smith JS, Nguyen TT, Nguyen HN, Ashley R, Snijders PJ, Meijer CJ, Munoz N, Parkin DM and Franceschi S: Human papillomavirus infection among women in South and North Vietnam. Int J Cancer 104: 213-220, 2003.

11 Tran LT, Tran LT, Bui TC, Le DT, Nyitray AG, Markham CM, Swartz MD, Tran CB and Hwang LY: Risk factors for high-risk and multi-type Human Papillomavirus infections among women in Ho Chi Minh City, Vietnam: a cross-sectional study. BMC Women's Health 15: 16, 2015.

12 Charan $\mathrm{J}$ and Biswas T: How to calculate samples size for different study designs in medical research. Indian J Psychol Med 35: 121-126, 2013.

13 Lee EH, Um TH, Chi HS, Hong YJ and Cha YJ: Prevalence and distribution of human papillomavirus infection in Korean women as determined by restriction fragment mass polymorphism assay. J Korean Med Sci 27: 1091-1097, 2012.

14 Kjaer SK, Breugelmans G, Munk C, Junge J, Watson M and Iftner T: Population-based prevalence, type- and age specific distribution of an HPV vaccination program in Denmark. Int J Cancer 123: 1864-1870, 2008.

15 Clifford GM, Gallus S, Herrero R, Munoz N, Snijders PJF, Vaccarella S, and IARC HPV Prevalence Surveys Study Group: Worldwide distribution of human papillomavirus types in cytologically normal women in the International Agency for Research on Cancer HPV prevalence surveys: a pooled analysis. Lancet 366: 991-998, 2005.

16 Rousseau MC, Abrahamowicz M, Villa LL, Costa Mc, Rohan TE and Franco EL: Predictors of cervical coinfection with multiple human papillomavirus types. Cancer Epidemiol Biomarkers Prev 12: 1029-1037, 2003.

17 Ramanakumar AV, Naud P, Roteli-Martins CM, de Carvalho NS, de Borba PC, Teixeira JC, and HPV-007 Study Group: Incidence and duration of type-specific human papillomavirus infection in high-risk HPV-naïve women: results from the control arm of a phase II HPV-16/18 vaccine trial. BMJ Open 6: e01137, 2016.

18 Zeng Z, Yang H, Li Z, He X, Griffith CC, Chen X, Guo X, Zheng $\mathrm{B}$, Wu $\mathrm{S}$ and Zhao $\mathrm{C}$ : Prevalence and genotype distribution of HPV infection in China: analysis of $51345 \mathrm{HPV}$ 
genotyping results from China's largest CAP certified laboratory. J Cancer 7: 1037-1043, 2016.

19 Hildesheim A, Gravitt P, Schiffman MH, Kurman RJ, Barnes W, Jones S, Tchabo JG, Brinton LA, Copeland C, Epp J, Janet BS and Manos MM: Determinants of genital human papilloma virus infection in low-income women in Washington, D.C. Sex Transm Dis 20: 279-285,1993.

20 Winer RL, Lee SK, Hughes JP, Adam DE, Kiviat NB and Koutsky LA: Genital human papillomavirus infection: rates and risk factors in a cohort of female university students. Am J Epidemiol 157: 218-226, 2003.

21 Ho GYF, Bierman R, Beardsley L, Chang CJ and Burk RD: Natural history of cervico-vaginal papillomavirus infection in young women. N Engl J Med 338: 423-428, 1998.

22 Winer RL, Hughes JP, Feng Q, O'Reilly S, Kiviat NB, Holmes KK and Koutsky LA: Condom use and the risk of genital human papillomavirus infection in young women. $\mathrm{N}$ Engl J Med 354: 2645-2654, 2006.
23 Veldhuijzen NJ, Snijders PJ, Reiss P, Meijer CJ and van de Wijgert JH: Factors affecting transmission of mucosal human papillomavirus. Lancet Infect Dis 10: 862-874, 2010.

24 Ramamurthi R, Rajarajeswari S and Ranjani Devi R: Comparison of knowledge, attitude and practices regarding cervical cancer screening and HPV vaccination between employed and unemployed women: a cross-sectional study. Int J Med Sci Clin Invest 2: 624-632, 2015.

Received December 14, 2016

Revised January 27, 2017

Accepted February 1, 2017 